\title{
Statistical Properties of a Polymer Chain in the Environment with Low Concentration of Nanoparticles
}

\author{
Dessalegne A. Tsehay and Meng-Bo LuO* \\ Department of Physics, Zhejiang University, Hangzhou 310027, China \\ (Received September 23, 2017; in final form November 16, 2017)
}

\begin{abstract}
We have investigated the statistical properties of polymer in the environment with low concentration of nanoparticles by using large-scale molecular dynamics simulations. The scaling law for the mean square radius of gyration was examined and simulation results for the polymer lengths $64 \leq N \leq 144$ yielding a reasonably accurate value of the Flory exponent $\nu=0.58$ at weak polymer-nanoparticle interaction $\varepsilon_{\mathrm{PN}}$. Within the same range of $N$, the mean asphericity of the chain is independent of $N$. We found that the polymer behaves like a self-avoiding walk chain at small $\varepsilon_{\mathrm{PN}}$ and a compact sphere at large $\varepsilon_{\mathrm{PN}}$. The results are attributed to the increase in the contact between polymer and nanoparticles with increasing $\varepsilon_{\mathrm{PN}}$. Normal diffusions of polymer are always observed at whatever $\varepsilon_{\mathrm{PN}}$ and size and concentration of nanoparticles. Our result shows that the normal diffusion behavior of polymer is independent of polymer's state even though there is a phase transition from a desorbed polymer phase at small $\varepsilon_{\mathrm{PN}}$ to an adsorbed polymer phase at large $\varepsilon_{\mathrm{PN}}$.
\end{abstract}

DOI: 10.12693/APhysPolA.133.1274

PACS/topics: diffusion, conformation, polymer chain, nanoparticles, molecular dynamics

\section{Introduction}

Diffusivity of polymers in random disordered media is of practical interest for application including enhancing oil recovery, drug delivery, gel electrophoresis, exclusion chromatography and DNA sorting [1]. Practical examples are the diffusion of macromolecules in crowded living cells [2] and gels [3], and the transport of proteins in intracellular environments [4]. The living cell is a crowded environment filled with particles smaller or larger than protein or DNA chain. The large crowding in the cellular environment affects the diffusivity of both small and large molecules and the motions of molecules are complicated [5]. In the similar way, the translocation of polymer through nanopore, a ubiquitous process in chemical and biological systems, was also influenced by crowding [6]. A polymer nanocomposite (PNC) is another example where polymer is in an environment with nanoparticles (NPs). Because of advantageous electrical, optical, or mechanical properties [7, 8], there has been considerable interest in using NPs to enhance polymers' physical properties relative to the native polymers $[9,10]$. For biomedical application, gold NPs are required to be coated by peptide chains [11]. It was found the dynamics of peptide chains was influenced significantly [11].

The behavior of a single polymer chain in a disordered medium has served as a model system to study the fundamental aspect of the effect of NPs on polymer chains [12]. A central question for polymer in the environment with NPs is to what extent the static and dynamic properties of polymers are influenced by NPs. Several computational studies in this area shed light on the issue of glass transition temperature in NP filled polymer melts,

*corresponding author; e-mail: luomengbo@zju.edu.cn as well as the effect of surface interactions on polymer diffusivity and viscosity [13]. The existence of NPs can change the dynamics of polymers and therefore shifts the glass transition temperature [14] and slows down the diffusion of polymer chains [15]. Recently, the dynamics of polymer melts and concentrated polymer solutions have been studied intensively by experiments $[16,17]$, theory and computer simulations [18-21]. It was found that the properties of polymers would be influenced by many parameters, such as size, shape, and concentration of NPs and interaction strength between polymer and NP [10]. Polymer chains can be expanded, collapsed, or even be unaffected by NPs, depending on the polymer-NP interaction and size of NPs $[16,22]$. The viscosity of polymers can be also influenced by a loading of NPs. It was found that the melt viscosity of poly (butylene succinate) (PBS) could be enhanced significantly by three orders of magnitude by loading a small-volume fraction of spherical NPs [23]. Molecular dynamics (MD) simulations performed in the regime of optimal NP dispersion and strong polymer-NP interactions showed that smaller NPs possessed better reinforcing properties, leading to tougher PNCs [18]. Thus, the properties of polymer at low concentration of NP's are also an interesting topic.

The effect of periodically distributed and randomly distributed NPs on the polymer's static and dynamic properties was studied by using the Monte Carlo (MC) simulations [21]. In those studies, NPs are immobile to mimic NPs in real polymer materials [10]. In this paper, we extend our study to the effect of mobile NPs on polymer properties since NPs in living cells or in polymer melts are mobile. We take the polymer-NP interaction and the size and concentration of NPs into account and investigate the effect of them on the statistical and dynamical properties of polymer. By using MD simulation, we find that the polymer undergoes a phase transition from a desorbed polymer phase at weak polymer-NP interac- 
tion to an adsorbed polymer phase at strong polymer-NP interaction, while the diffusion of polymer is always normal irrespective of the state of polymer. However, the diffusion coefficient is dependent on the polymer-NP interaction and the size and concentration of NPs, i.e. the state of polymer.

\section{Model and simulation method}

MD simulations are performed using Large Scale Atomic/Molecular Massively Parallel Simulator (LAMMPS) software [24]. LAMMPS is a widelyused software simulator for molecular dynamics. The simulation is performed in a cubic system with side length $L$, and is chosen to be larger than the average chain size (represented by the mean end-to-end distance $R)$. We have checked the finite-size effect and found it is not noticeable when $L>2 R$. Periodic boundary conditions (PBC) are adopted in all three directions. There is one single polymer chain and a fixed number of identical NPs in the simulation system. The polymer chain of length $N$ is constructed by $N$ identical monomers. The $\mathrm{NP}$ is a spherical particle with a diameter $\sigma_{N P}$. The units of length, energy, and mass are $\sigma=1, k_{\mathrm{B}} T=1$, and $m=1$, respectively. The reduced unit for time is described as $\tau=\sqrt{\frac{m \sigma^{2}}{\varepsilon}}$. Here $\sigma$ is the diameter of monomer, $k_{\mathrm{B}}$ is the Boltzmann constant, $T$ is the temperature, and $m$ is mass of monomer. The mass of NPs is assumed to be proportional to the volume of NPs as $m_{\mathrm{NP}}=\left(\sigma_{\mathrm{NP}} / \sigma\right)^{3} m$.

The bonded monomers in the polymer chain is connected by a finitely extensible nonlinear elastic (FENE) potential

$$
\begin{gathered}
V_{\mathrm{FENE}}(b)=-\frac{1}{2} K R_{0}^{2} \ln \left[1-\left(\frac{\mathrm{b}}{R_{0}}\right)^{2}\right] \\
+4 \varepsilon_{\mathrm{B}}\left[\left(\frac{\sigma}{b}\right)^{12}-\left(\frac{\sigma}{b}\right)^{6}\right]+\varepsilon_{\mathrm{B}}
\end{gathered}
$$

with $K=30, R_{0}=1.5 \sigma, \varepsilon_{\mathrm{B}}=1$, and $\sigma=1$. Here $b$ is the bond length. The bond length $b$ in the FENE term extends to $R_{0}$, the maximum extent of the bond. The second Lennard-Jones (LJ) term is cutoff at $b_{c}=2^{1 / 6} \sigma$. The first term is attractive, while the $2 \mathrm{nd} \mathrm{LJ}$ term is repulsive. The combined outcome of the two terms maintains an equilibrium bond length and prevents bonds from crossing each other. The energetic interaction between any pair of non-bonded monomers is modeled by a truncated and shifted LJ potential

$$
V(r)=4 \varepsilon_{\mathrm{PP}}\left[\left(\frac{\sigma}{r}\right)^{12}-\left(\frac{\sigma}{r}\right)^{6}\right]
$$

for $r\left\langle r_{c}=2.5 \sigma\right.$ and the potential is shifted to zero at $r_{c} . V(r)=0$ for $r>r_{c}$. Here $r$ is the distance between two non-bonded monomers. We set a weak interaction strength $\varepsilon_{P P}=0.2$ for the polymer with the intention that the polymer behaves as a self-avoiding walk (SAW) chain at $k_{\mathrm{B}} T=1$.
The NPs are modeled as a sphere with the diameter $\sigma_{\mathrm{NP}} \geq \sigma$. For the case $\sigma_{\mathrm{NP}} \geq \sigma$, the interaction between monomer and NP is simply described via a modified LJ potential

$$
V(r)=4 \varepsilon_{\mathrm{PN}}\left[\left(\frac{\sigma}{r-\Delta}\right)^{12}-\left(\frac{\sigma}{r-\Delta}\right)^{6}\right]
$$

with $\Delta=\frac{\sigma+\sigma_{\mathrm{NP}}}{2}-\sigma[18]$. In this system the long-ranged polymer-NP attraction is considered by setting the cutoff distance $r_{c}=\Delta+2.5 \sigma$. The potential is also shifted to zero at $r_{c}$. In the simulations, we change the polymerNP interaction strength $\varepsilon_{\mathrm{PN}}$ and the NP's size $\sigma_{\mathrm{NP}}$ to study the effect of NPs. The interaction between two NPs is set as purely repulsive LJ potential, which is of the same form as equation 3 but with $\Delta=\sigma_{\mathrm{NP}}-\sigma$ and cutoff distance $r_{c}=\Delta+2^{1 / 6} \sigma$. The interaction strength parameter for the NP-NP interaction is set as $\varepsilon_{\mathrm{NN}}=1$.

The simulation system contains one polymer chain with $N$ monomers and $N_{\mathrm{NP}}$ NPs. The move of monomers and NPs obeys the Langevin equation

$$
m \frac{\mathrm{d}^{2} \boldsymbol{r}}{\mathrm{d} t^{2}}=-\nabla V-\eta \boldsymbol{v}+\boldsymbol{F}_{r}
$$

Here $\eta$ is the frictional coefficient. The systems are run in the canonical (NVT) ensemble with the Langevin mechanism with a frictional coefficient of $\eta=0.1$ to maintain the temperature of system. In the NVT ensemble, amount of substance $(N)$, volume $(V)$ and temperature $(T)$ are conserved. The thermal random force $\boldsymbol{F}_{r}$ is a Gaussian white noise with zero mean, rewarding the fluctuation-dissipation relation

$$
\left\langle\boldsymbol{F}_{i}(t) \cdot \boldsymbol{F}_{j}\left(t^{\prime}\right)\right\rangle=6 \eta k_{\mathrm{B}} T \delta_{i j} \delta\left(t-t^{\prime}\right) .
$$

The simulations are performed with the following two main steps: an equilibrium step and a statistics step. The system is at first equilibrated for a sufficient time, much longer than the relaxation time of the polymer chain. Afterwards we run a long time for the statistics. The end of the equilibrium step is set as the initial time $t=0$ for the statistics. The statistical size of the polymer chain is described by the mean square radius of gyration

$$
\left\langle R_{G}^{2}\right\rangle=\frac{1}{N} \sum_{i=1}^{N}\left(\boldsymbol{r}_{i}-\boldsymbol{r}_{c m}\right)^{2} .
$$

Here $\boldsymbol{r}_{c m}$ is the position vector of the center of mass of polymer chain. The diffusion is characterized by the mean-square displacement (MSD) of the center of mass

$$
\left\langle\Delta r^{2}(t)\right\rangle=\left\langle\left[\boldsymbol{r}_{c m}(t)-\boldsymbol{r}_{c m}(0)\right]^{2}\right\rangle .
$$

Here $\boldsymbol{r}_{c m}(t)$ is the position vector of the center of mass of polymer chain at time $t$ and $\boldsymbol{r}_{c m}(0)$ at initial time $t=0$. \langle\rangle denotes the statistical average over different independent samples. In this work, more than 500 samples are simulated for obtaining the average values.

\section{Results and discussions}

Figure 1 presents the dependence of the mean square radius of gyration $\left\langle R_{G}^{2}\right\rangle$ on the polymer-NP interaction strength $\varepsilon_{\mathrm{PN}}$ for different concentrations of NPs. 


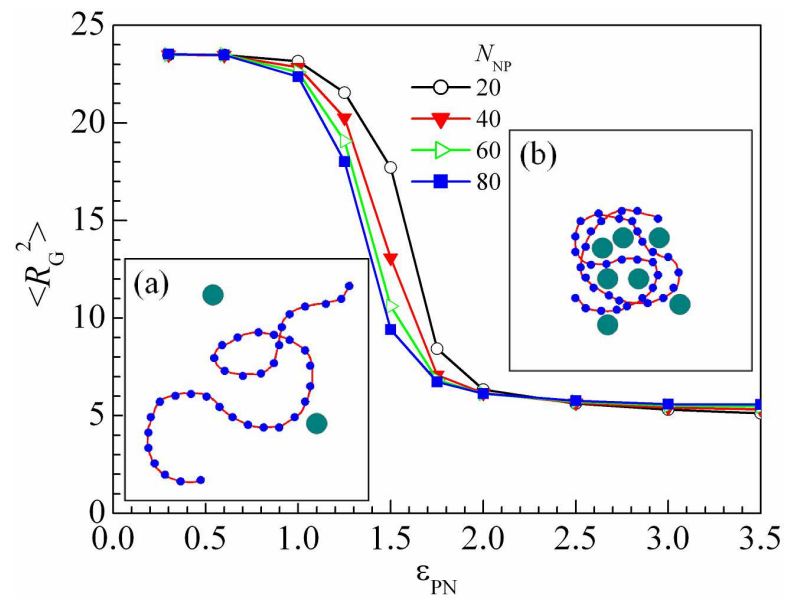

Fig. 1. Plot of the mean square radius of gyration, $\left\langle R_{G}^{2}\right\rangle$, as a function of the polymer-NP interaction $\varepsilon_{\mathrm{PN}}$ for polymer in the environment with number of NPs $N_{\mathrm{NP}}=20,40,60$, and 80 . The insets (a) and (b) show the sketches of polymer chain at weak and strong polymer-NP interactions, respectively. Parameters: polymer length $N=64$, size of NP $\sigma_{\mathrm{NP}}=1$, and system size $L=30$.

The polymer length $N=64$ and the size of NP $\sigma_{\mathrm{NP}}=1$ are used in simulations. Here the concentration of NPs is defined as:

$$
C_{\mathrm{NP}}=\frac{N_{\mathrm{NP}} V_{\mathrm{NP}}}{V} \text {. }
$$

Here $V_{\mathrm{NP}}=\frac{1}{6} \pi \sigma_{\mathrm{NP}}^{3}$ is the volume of single $\mathrm{NP}$ and $V=L^{3}$ is the volume of the system. $C_{\mathrm{NP}}$ is linearly proportional to the number of NPs, $N_{\mathrm{NP}}$. The size of the simulation system is $L=30$ for polymer with $N=64$ ( $R$ is about 12 ). At weak interaction strength $\varepsilon_{\mathrm{PN}}$ less than 1 , we find that $\left\langle R_{G}^{2}\right\rangle$ is roughly independent of the polymer-NP interaction and keeps a high value. The reason is that only a few monomers are in contact with NPs at weak polymer-NP interaction. For example, we find that about $7 \%$ of monomers, averaged in the simulation time, are in contact with NPs at $\varepsilon_{\mathrm{PN}}=1$ for the case of $N_{\mathrm{NP}}=20$. Thus the conformational properties of the polymer are roughly not or weakly affected by NPs as shown in the inset (a) of Fig. 1, whereas at intermediate $\varepsilon_{\mathrm{PN}}$ starting from $\varepsilon_{\mathrm{PN}}=1.0$ to $\varepsilon_{\mathrm{PN}}=2.0$, \rangle $\left.R_{G}^{2}\right\rangle$ drops significantly to a low value. This means that the attracting NPs pull polymer together, resulting in a more compact conformation of the polymer chain. We find about $82 \%$ of monomers are in contact with NPs at $\varepsilon_{\mathrm{PN}}=2$ for the case of $N_{\mathrm{NP}}=20$. In this transitional interaction regime, we find that $\left\langle R_{G}^{2}\right\rangle$ decreases faster at larger concentration of NPs. Finally, at strong polymer-NP interaction strength $\varepsilon_{\mathrm{PN}}$ larger than 2 , the polymer is in the compact globule state as shown in the inset (b) of Fig. 1 and the value of $\left\langle R_{G}^{2}\right\rangle$ roughly keeps a constant value independent of the interaction strength and concentration of NPs.

The main result of Fig. 1 can be summarized as that there is a phase transition from extended coil state of polymer chain at weak polymer-NP attraction to compact globule states at strong polymer-NPs interaction and the transition is roughly independent of the concentration of NPS.

We have also calculated the instantaneous shape of polymer. The shape of polymer is usually described by the asphericity parameter $A$, which is defined as:

$$
A=\sum_{i>j}^{3}\left(L_{i}^{2}-L_{j}^{2}\right)^{2} / 2\left(\sum_{i=1}^{3} L_{i}^{2}\right)^{2}
$$

in three-dimensional (3D) space. Here $L_{1}^{2}, L_{2}^{2}$ and $L_{3}^{2}$ $\left(L_{1}^{2} \leq L_{2}^{2} \leq L_{3}^{2}\right)$ are three eigenvalues of a 3D radiusof-gyration tensor. The relation between the three eigenvalues and the square radius of gyration $R_{G}^{2}$ is simply as $R_{G}^{2}=L_{1}^{2}+L_{2}^{2}+L_{3}^{2}$. The mean asphericity parameter $\langle A\rangle$ varies from 0 for spherically symmetric chain conformations, to 1 for long rods. While for linear SAW polymers, $\langle A\rangle$ is about 0.43 for long polymers [25]. For $N=64$ in the environment with $20 \mathrm{NPs}$, we find $\langle A\rangle=0.43$ at $\varepsilon_{\mathrm{PN}}=0.6$ and $\langle A\rangle=0.42$ at $\varepsilon_{\mathrm{PN}}=1$, indicating that the polymer behaves like a SAW-like chain at weak $\varepsilon_{\mathrm{PN}}$. However, it drops significantly to 0.09 at $\varepsilon_{\mathrm{PN}}=2$ and 0.04 at $\varepsilon_{\mathrm{PN}}=3$, close to the value of a compact sphere. Thus the polymer looks like a sphere at large $\varepsilon_{\mathrm{PN}}$.

Similar results are found for other polymer lengths as shown in Fig. 2a. It seems that the intermediate interaction region where $\left\langle R_{G}^{2}\right\rangle$ drops obviously is roughly independent of the polymer length. Figure $2 \mathrm{~b}$ presents a $\log -\log$ plot of $\left\langle R_{G}^{2}\right\rangle$ versus polymer length $N$ at different attraction interactions. The power-law relation $\left\langle R_{G}^{2}\right\rangle \sim N^{2 v}$ with $\nu \approx 0.58$ is observed at small $\varepsilon_{\mathrm{PN}}$. The value of Flory exponent $\nu \approx 0.58$ indicates that the polymer chain behaves as a self-avoiding walk chain. Conversely, at large $\varepsilon_{\mathrm{PN}}$, the behavior of short polymers seems to be a compact globule since the exponent $\nu$ tends to be $1 / 3$. At large $\varepsilon_{\mathrm{PN}}$ polymer is aggregated by the attraction of NPs. However the aggregation of polymer becomes weak with the increase in polymer length since the number of NPs is limited in the system. Therefore, we find the scaling behavior deviates from $1 / 3$ for long polymers.

Figure 3 presents the conformation size $\left\langle R_{G}^{2}\right\rangle$ of a polymer chain as a function of NP's size, $\sigma_{\mathrm{NP}}$, for different polymer-NP interactions. The dependence of $\left\langle R_{G}^{2}\right\rangle$ on $\sigma_{\mathrm{NP}}$ is dependent on the polymer-NP interaction strength $\varepsilon_{\mathrm{PN}}$. At weak polymer-NP interaction $\left\langle R_{G}^{2}\right\rangle$ is roughly independent of $\sigma_{\mathrm{NP}}$, indicating that the polymer chain is not affected by the NPs. In the intermediate interaction regime near $\varepsilon_{\mathrm{PN}}=1.5,\left\langle R_{G}^{2}\right\rangle$ decreases at first and then increases steadily as $\sigma_{\mathrm{NP}}$ increases. At last $\left\langle R_{G}^{2}\right\rangle$ increases with increase of $\sigma_{\mathrm{NP}}$ at strong $\varepsilon_{\mathrm{PN}}$. The reason is that polymer and NPs are aggregated together at strong polymer-NP attraction. $\left\langle R_{G}^{2}\right\rangle$ is almost saturated when the polymer-NP interaction is strong.

The diffusive behavior of the polymer in the environment with NPs is also studied. The MSD of a single polymer defined by Eq. (7) is calculated. As an example, Fig. 4 shows log-log plot of the time dependant of MSD 

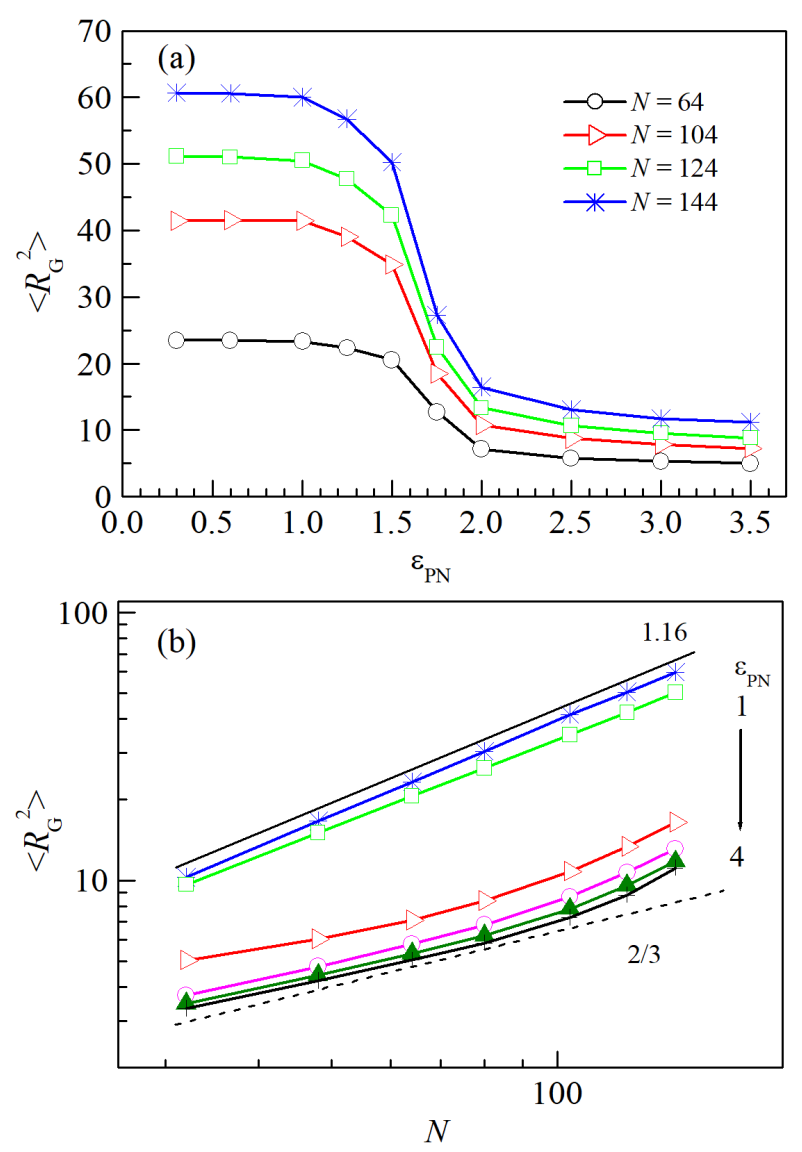

Fig. 2. (a) Plot of mean square radius of gyration $\left\langle R_{G}^{2}\right\rangle$ versus polymer-NP interaction $\varepsilon_{\mathrm{PN}}$ for various polymer chain length $N$, (b) $\log$-log plot of $\left\langle R_{G}^{2}\right\rangle$ versus $N$ for different $\varepsilon_{\mathrm{PN}}=1,1.5,2,2.5,3$, and 3.5 (from top to bottom). Parameters: size of NP $\sigma_{\mathrm{NP}}=1$, number of NPs is $N_{\mathrm{NP}}=10$, and system size $L=30$. Solid and dashed straight lines in (b) show the slope 1.16 and $2 / 3$, respectively.

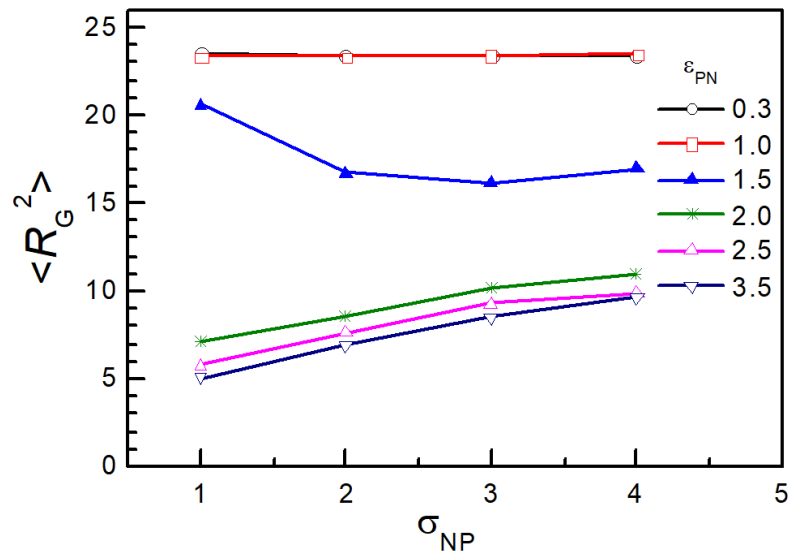

Fig. 3. Plot of mean square radius of gyration $\left\langle R_{G}^{2}\right\rangle$ as a function of NP's size $\sigma_{\mathrm{NP}}$ for various polymer-NP interactions $\varepsilon_{\mathrm{PN}}=0.3,1.0,1.5,2.0,2.5$, and 3.5 (from top to bottom). Parameters are: polymer length $N=$ 64 , number of NPs is $N_{\mathrm{NP}}=10$, and system size $L=30$. for different concentrations of NPs for the polymer length $N=64$. We find polymers of length $N=64,104,124$, 144 have similar behavior, so here we only present results for $N=64$. We find the polymer chain has the same diffusive behaviors for all concentration of NP's, and normal diffusion $\left\langle\Delta r^{2}\right\rangle \sim t$ is observed at long time scale. Such kind normal diffusion was also observed by MC simulations for the polymer in a crowded environment with mobile NPs at whatever attraction strength of NPs [12]. Some experiments also pointed out the normal diffusion of polymer in systems with NPs [10]. However, the diffusion was found abnormal for polymer in system with immobile NPs at strong attraction between polymer and NPs [21].

Moreover, we find the normal diffusion of polymer at long time scale for different $\varepsilon_{\mathrm{PN}}, \sigma_{\mathrm{NP}}$, and $C_{\mathrm{NP}}$. Thus we estimate the translational diffusion coefficient $D$ of polymer via the linear fit from the relation $\left\langle\Delta r^{2}\right\rangle=6 D t$ at long time scale. However the translational diffusion coefficient $D$ is dependent on these ingredients.

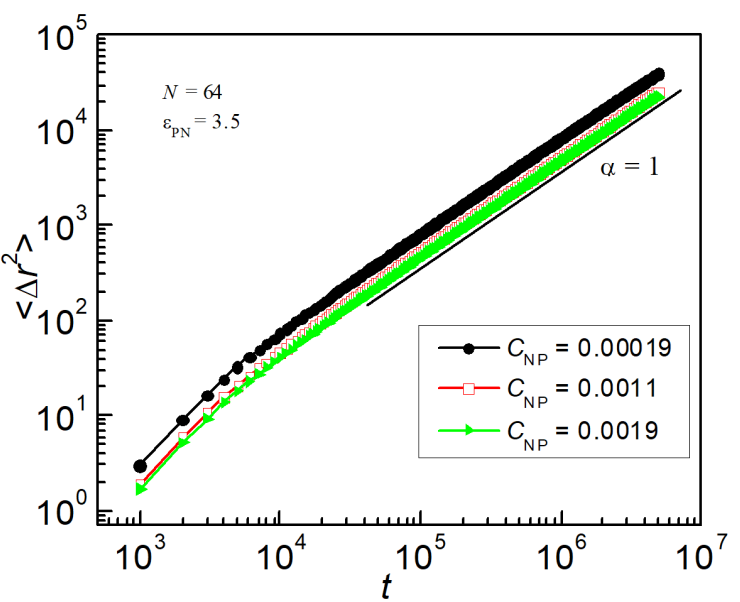

Fig. 4. Log-log plot of the mean square displacement of the center of mass of the polymer $\left\langle\Delta r^{2}\right\rangle$ as a function of simulation time for different concentration of NP's $\mathrm{C}_{\mathrm{NP}}$. The solid line represents the line of best fit with the slope $\alpha=1$.

Figure 5 shows the variation of the diffusion coefficient $D$ of polymer with the polymer-NP interaction $\varepsilon_{\mathrm{PN}}$ for different NP's sizes. The dependence of $D$ on $\varepsilon_{\mathrm{PN}}$ is dependent on the NP's size $\sigma_{\mathrm{NP}}$. The polymer length $N=64$ and the number of NPs $N_{\mathrm{NP}}=10$ are fixed in the simulations. We find that $D$ keeps a high value and it is roughly independent of the size of NP at weak interaction strength $\varepsilon_{\mathrm{PN}}<1.0$. However, $D$ decreases obviously with increasing $\varepsilon_{\mathrm{PN}}$ in the intermediate region from $\varepsilon_{\mathrm{PN}}=1.0$ to $\varepsilon_{\mathrm{PN}}=2.0$. The decrease in $D$ is more obvious for larger NPs. In this intermediate interaction region, NPs attract the polymer leads to a low $D$ as well as a small $\left\langle R_{G}^{2}\right\rangle$ (Fig. 1). As the NP's size increases, NPs have more attracting surface to attract the polymer chain, thus $D$ decreases faster at larger NP's size. Finally, at strong interaction strength $\varepsilon_{\mathrm{PN}}>2.0$, due to the firm contact between polymer and NPs, $D$ drops to a low value 
and becomes roughly independent of $\varepsilon_{\mathrm{PN}}$. We find that the behavior of $D$ is similar to that of $\left\langle R_{G}^{2}\right\rangle$ as shown in Fig. 1a. The results show that the diffusion of polymer is dependent on the conformation of polymer, in agreement with previous Monte Carlo simulation. It was found that the diffusion coefficient of polymer was low for a compact conformation in solution or on flat surface [26].

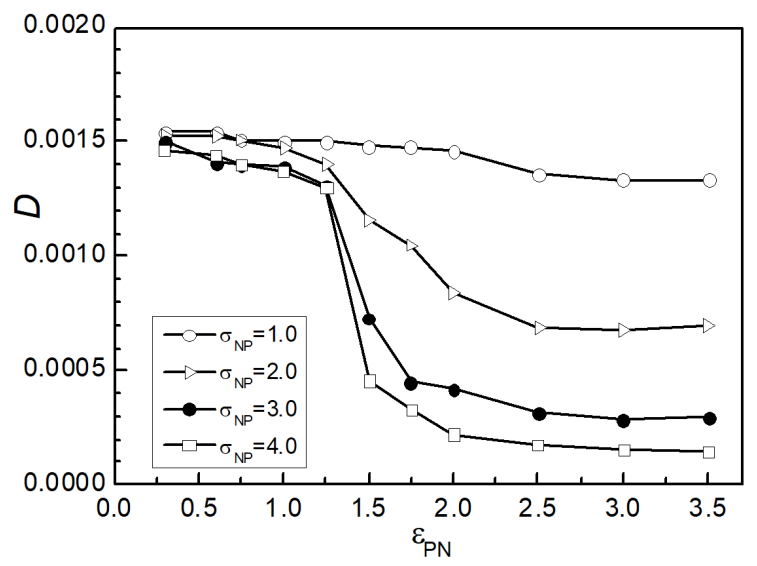

Fig. 5. Diffusion coefficient of polymer chain $D$ as, a function of polymer-NPs interaction $\varepsilon_{\mathrm{PN}}$ for various filler sizes. Simulation parameters are: chain length $N=64$, number of NPs is $N_{\mathrm{NP}}=10$, and system size $L=30$.

We have also checked the effect of NP's size on the diffusive property of polymer chain at the same concentration of NPs. That is, we change $\sigma_{\mathrm{NP}}$ but keeping $N_{\mathrm{NP}} \sigma_{\mathrm{NP}}^{3}$ constant. For the case $N_{\mathrm{NP}} \sigma_{\mathrm{NP}}^{3}=640$, we have compared the diffusivity of polymer for two systems: (1) $N_{\mathrm{NP}}=640$ and $\sigma_{\mathrm{NP}}=1$ and $(2) N_{\mathrm{NP}}=10$ and $\sigma_{\mathrm{NP}}=4$ . We find that the diffusion coefficient $D$ of system (2) with larger $\sigma_{\mathrm{NP}}$ is smaller than that of system (1) at large $\varepsilon_{\mathrm{PN}}>1.5$. For example, at $\varepsilon_{\mathrm{PN}}=3, D=0.00056$ for the system (1) while $D=0.00016$ for the system (2). The drop of $D$ for large $\sigma_{\mathrm{NP}}$ is always found in our simulations. We therefore we conclude that larger NPs are more effective to decrease the diffusion of polymer.

The dependence of $D$ on the size of NPs is plotted in Fig. 6 for the polymer with length $N=64$ at different polymer-NP interactions. At weak polymer-NP interactions $\varepsilon_{\mathrm{PN}}=0.6$ and $1.0, D$ decreases slightly with increasing $\sigma_{\mathrm{NP}}$. However, we find that $D$ decreases with increasing $\sigma_{\mathrm{PN}}$ for $\varepsilon_{\mathrm{PN}}$ in the intermediate interaction region from 1.0 to 2.0 or in the strong interaction region $\varepsilon_{\mathrm{PN}}>2.0$. At strong polymer-NP interaction, the motion of the polymer chain is expected to be slowed down due to the firm contact between polymer and NPs. The mass of NP increases with $\sigma_{\mathrm{PN}}$, the diffusion of NP decreases with $\sigma_{\mathrm{PN}}$, too. At $\varepsilon_{\mathrm{PN}}>1$, polymer starts to be contacted with NPs and the diffusion of the polymer is influenced strongly by the random diffusion of NPs. Therefore, the diffusion of polymer is slowed and $D$ decreases with increasing $\sigma_{\mathrm{PN}}$. While at large $\varepsilon_{\mathrm{PN}}>2$, polymer is firmly contacted with NPs and thus the diffusion of the polymer is further decreased.

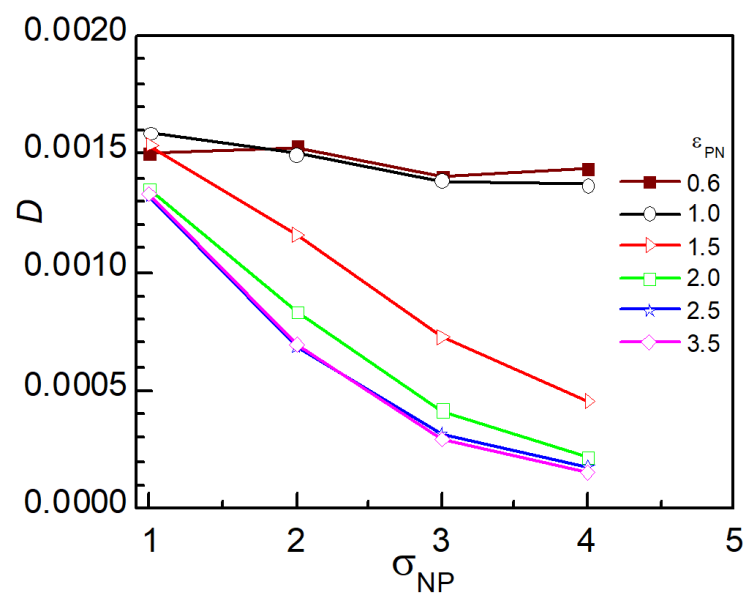

Fig. 6. Plot of diffusion coefficient $D$ of polymer chain as a function of NP's size $\sigma_{\mathrm{PN}}$ for various polymer-NP interactions $\varepsilon_{\mathrm{PN}}$, Simulation parameters are: polymer length $N=64$, number of NPs is $N_{\mathrm{NP}}=10$, and system size $L=30$.

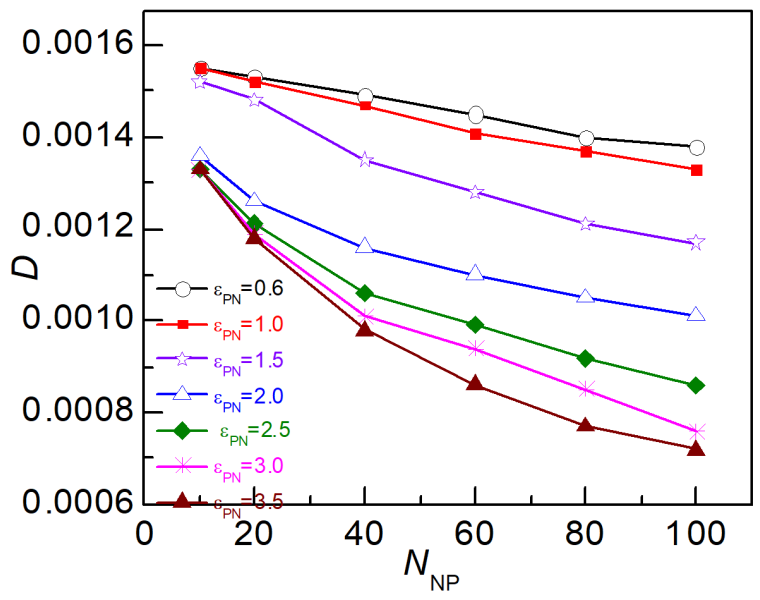

Fig. 7. Plot of diffusion coefficient of polymer chain $D$ as a function of the number of NPs $N_{N P}$ for various polymer-NP interactions $\varepsilon_{\mathrm{PN}}$. Simulation parameters are: polymer length $N=64$, NP's size $\sigma_{\mathrm{PN}}=1$, and system size $L=30$.

We have also studied the influence of the concentration of NPs on the polymer diffusivity. The results of the diffusion coefficient as a function of NP's concentration for different polymer-NP interaction strengths are presented in Fig. 7. For all cases, $D$ decreases with increase of the NP's concentration. The result is consistent with lattice MC simulation [27]. Experiments on polymer diffusivity in crowded PNC also found that the diffusion coefficient decreases with increase of the NP's concentration [10]. Here our results show that such a decrease in $D$ can be observed for all polymer-NP interactions, and $D$ decreases more obviously for larger $\varepsilon_{\mathrm{PN}}$.

Our simulation results on the polymer's statistical size and diffusivity reveal the important role of the contact between polymer and NPs. The contact between polymer and NPs is dependent on the polymer-NP inter- 
action. To understand the behavior of polymer in the environment with NPs, we have investigated the effect of NP's property on the contact between polymers with NPs. From the definition of the LJ potential between polymer and NP as shown in Eq. (3), the lowest energy is at $r=\Delta+2^{1 / 6}$. We then define a contact monomer in this work when the monomer locates near NPs with a distance $r<\Delta+1.5$, i.e. the distance between the center of monomer and surface of NP is less than 1.0. The number of monomers in contact with NPs, $N_{\text {cont }}$, has been counted in the simulations.

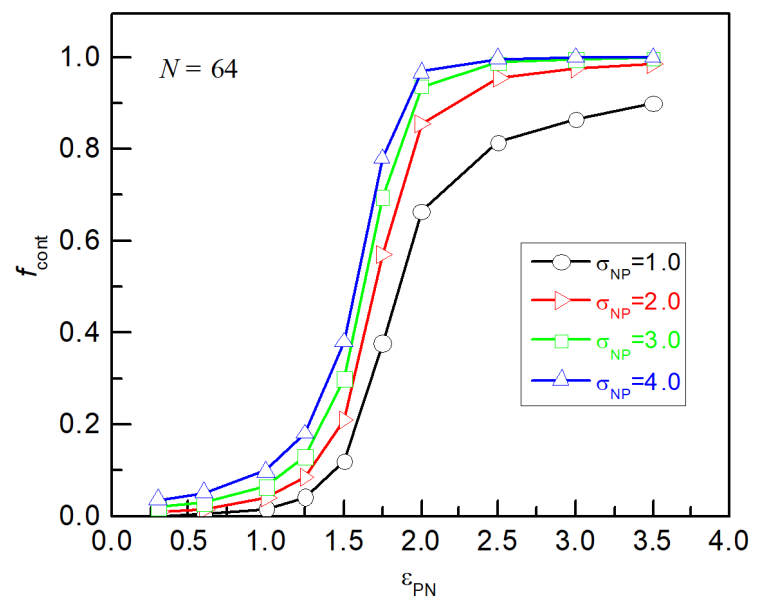

Fig. 8. Plot of the fraction of contacted monomers with NPs, $f_{\text {cont }}$, as a function of polymer-NP interaction $\varepsilon_{\mathrm{PN}}$ for different NP's sizes. Simulation parameters are: chain length $N=64$, number of NPs is $N_{\mathrm{NP}}=10$, and system size $L=30$.

Figure 8 presents the dependence of the fraction of contacted monomers, $f_{\text {cont }}=N_{\text {cont }} / N$, with polymer-NP interaction $\varepsilon_{\mathrm{PN}}$ for different NP's sizes for the polymer chain with length $N=64$. We find that $f_{\text {cont }}$ increases gradually with $\varepsilon_{\mathrm{PN}}$ for all NP's sizes. At weak polymerNP interaction strength only a few monomers are in contact with NPs. The value $f_{\text {cont }}$ remains a low value for $\varepsilon_{\mathrm{PN}}=0.3,0.6,1.0$. In the intermediate region in between $\varepsilon_{\mathrm{PN}}=1.5$ and $\varepsilon_{\mathrm{PN}}=2.0$, the polymer started to be adsorbed on NPs. As a result, the curve grows up steeply. Furthermore, at strong interaction strength $\varepsilon_{\mathrm{PN}}>2.0$, polymer and NPs are aggregated firmly so that the fraction $f_{\text {cont }}$ remains at a large value and is roughly independent of the interaction strength. The fraction $f_{\text {cont }}$ increases with the size of NP, $\sigma_{\mathrm{NP}}$, since large NP can attract more monomers.

Figure 9 presents the dependence of the fraction of contacted monomers $f_{\text {cont }}$ on polymer length $N$ at weak polymer-NP interaction $\varepsilon_{\mathrm{PN}}=1.0$ and at strong one $\varepsilon_{\mathrm{PN}}=3.5$ at the environment with $N_{\mathrm{NP}}=10$ and $\sigma_{\mathrm{NP}}=1$. At $\varepsilon_{\mathrm{PN}}=1.0 f_{\text {cont }}$ is close to 0 , indicating polymer is roughly free. Thus we find polymer behaves as a random coil as presented in Fig. $2 \mathrm{~b}$ with the scaling exponent $\nu$ close to 0.58 for $\left\langle R_{G}^{2}\right\rangle \sim N^{2 v}$. Whereas at $\varepsilon_{\mathrm{PN}}=3.5, f_{\text {cont }}$ is close to 1 for short polymer, thus polymer is strongly in contact with NPs. The attractive
NPs aggregate polymer and thus polymer behaves like a sphere. However, $f_{\text {cont }}$ decreases with increase of $N$ at $\varepsilon_{\mathrm{PN}}=3.5$. The desorbed monomers of polymer will lead to an asymmetrical configuration deviated from a sphere. This is in agreement with the deviation of the scaling exponent $\nu$ from $1 / 3$ as shown in Fig. $2 \mathrm{~b}$ for long polymers. Our results clearly show that the effect of strong attractive NPs fades away with increase of polymer length for a fixed NP's concentration.

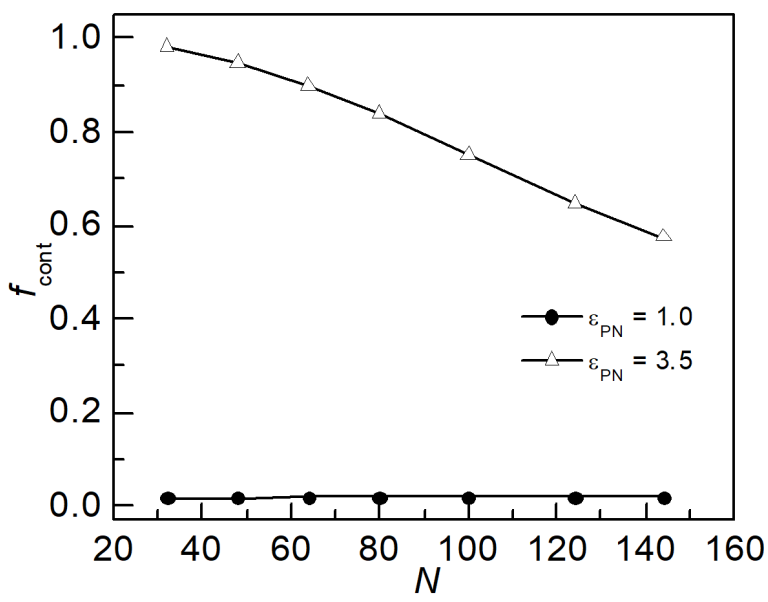

Fig. 9. Plot of the fraction of contacted monomers with NPs, $f_{\text {cont }}$, versus polymer chain length $N$ at polymer-NP interactions $\varepsilon_{\mathrm{PN}}=1.0$ and 3.5. Simulation parameters are: size of NP $\sigma_{\mathrm{NP}}=1$, number of NPs is $N_{\mathrm{NP}}=10$, and system size $L=30$.

\section{Conclusion}

We have studied the statistical and dynamical properties of the polymer in environment at low concentration of attractive spherical NP's. Both the statistical and dynamical properties of the polymer are dependent on interaction strength, the size and concentration of NPs. A phase transition from extended coil state of polymer chain at weak polymer-NP attraction to compact globule state at strong polymer-NPs interaction is observed. Normal diffusive behaviors of the polymer are observed for all simulation parameters. Larger NPs are more effective to decrease the diffusion coefficient of the polymer. The effect of NPs can be explained approximately from the contacting between NP and polymer chain.

The number of monomers in contacting with NPs increases with increase of the polymer-NP interaction strength. The increase in the contact number decreases the mean square radius of gyration $\left\langle R_{G}^{2}\right\rangle$ and the diffusion coefficient as well. The Flory exponent $\nu$ in the powerlaw relation $\left\langle R_{G}^{2}\right\rangle \sim N^{2 v}$ decreases from 0.58 at weak polymer-NP interaction to about $1 / 3$ at strong polymerNP interaction. The transition from extended coil state of polymer chain to compact globule state is equivalent to the transition from a desorbed state to an adsorbed state. The diffusion coefficient is roughly independent of the size of NP at weak polymer-NP interaction but decreases obviously with increase of the size of NP at strong 
polymer-NP interaction. However the diffusion coefficient always decreases with increase of the concentration of NPs. Our results show that the diffusive behavior of polymer does not change even though the state of polymer changes from extended coil to compact globule.

One of the application of our study is to understand the translocation process of polymer through nanopore in crowding environments. The translocation of polymers including DNA and protein through nanopores in membrane is influenced by NPs in the environment since living cell is crowded with macromolecules and other inclusions [28]. The interaction between polymers and obstacles was found to be an important role in the translocation of polymer through crowded channel [29] or into crowded environments $[27,30]$. Our results would be benefit for understanding the abnormal behaviors of polymer translocation in crowded environments.

\section{Acknowledgments}

This research was supported by the National Natural Science Foundation of China under Grant (No. 21574117).

\section{References}

[1] S.J.E. Lee, A.K. Chakraborty, J. Chem. Phys. 117, 10869 (2002).

[2] A.S. Verkman, Trends Biochem. Sci. 27, 27 (2002).

[3] R.M. Briber, X. Liu, B.J. Bauer, Science 268, 395 (1995).

[4] D.S. Banks, C. Fradin, Biophys. J. 89, 2960 (2005).

[5] I. Goychuk, V.O. Kharchenko, R. Metzler, PLoS ONE 9, e91700 (2014).

[6] A. Gopinathan, Y.W. Kim, Phys. Rev. Lett. 99, 228106 (2007).

[7] A.C. Balazs, T. Emrick, T.P. Russell, Science 314, 1107 (2006).

[8] Q.H. Zeng, A.B. Yu, G.D. Lu, Prog. Polym. Sci. 33, 191 (2008).
[9] L. Liu, P. Li, S.A. Asher, Nature 397, 141 (1999).

[10] S. Gam, J.S. Meth, S.G. Zane, C. Chi, B.A. Wood, K.I. Winey, N. Clarke, R.J. Composto, Soft Matter 8, $6512(2012)$.

[11] D. An, J. Su, J.K. Weber, X. Gao, R. Zhou, J. Li, J. Am. Chem. Soc. 137, 8412 (2015).

[12] M. Muthukumar, J. Non-Cryst. Solids 131, 654 (1991).

[13] F.W. Starr, T.B. Schroder, S.C. Glotzer, Macromolecules 35, 4481 (2002).

[14] F.W. Starr, T.B. Schroder, S.C. Glotzer, Phys. Rev. E 64, 021802 (2001).

[15] M. Vacatello, Macromolecules 34, 1946 (2001).

[16] S. Sen, Y.P. Xie, S.K. Kumar, H.C. Yang, A. Bansal, D.L. Ho, L. Hall, J.B. Hooper, K.S. Schweizer, Phys. Rev. Lett. 98, 128302 (2007).

[17] B.A. Paez-Sierra, D.M. Marulanda, Acta Phys. Pol. A 129, 1187 (2016).

[18] D. Gersappe, Phys. Rev. Lett. 89, 058301 (2002).

[19] M.A. Sharaf, J.E. Mark, Polymer 45, 3943 (2004).

[20] G.G. Vogiatzis, E. Voyiatzis, D.N. Theodorou, Eur. Polym. J. 47, 699 (2011).

[21] C.Y. Li, M.B. Luo, J.H. Huang, H. Li, Phys. Chem. Chem. Phys. 17, 31877 (2015).

[22] A. Sikorski, Acta Phys. Pol. A 107, 443 (2004).

[23] H. Li, Y. Luo, R. Qi, J. Feng, J. Zhu, Y. Hong, Z. Feng, P. Jiang, J. Appl. Polym. Sci. 132, 42193 (2015).

[24] S. Plimpton, J. Comput. Phys. 117, 1 (1995).

[25] M.B. Luo, J.H. Huang, J. Chem. Phys. 119, 2439 (2003).

[26] Q.H. Yang, M.B. Luo, Sci. Rep. 6, 37156 (2016).

[27] W.P. Cao, Q.B. Ren, M.B. Luo, Phys. Rev. E 92, 012603 (2015).

[28] E. Barkai, Y. Garini, R. Metzler, Phys. Today 65, 29 (2012).

[29] J.X. Chen, J.X. Zhu, Y.Q. Ma, J.S. Cao, EPL 106 18003 (2014).

[30] W.C. Yu, K.F. Luo, J. Am. Chem. Soc. 133, 13565 (2011). 